\title{
Systemic immune-inflammation index predicts prognosis of patients with advanced pancreatic cancer
}

\author{
Ke Zhang ${ }^{1,2}$, Yong-Qiang Hua 1,2, Dan Wang ${ }^{1,2}$, Lian-Yu Chen ${ }^{1,2}$, Cai-Jun Wu ${ }^{1,2}$, Zhen Chen 1,2, Lu-Ming Liu ${ }^{1,2}$ \\ and Hao Chen ${ }^{1,2^{*}}$
}

\begin{abstract}
Background: Systemic inflammation and immune dysfunction have been proved to be associated with cancer progression and metastasis in various malignancies. The aim of this retrospective study was to evaluate the prognostic significance of pre-treatment systemic immune-inflammation index (SII) in patients with advanced pancreatic cancer.

Methods: In total, 419 patients diagnosed with advanced pancreatic cancer, between January 2011 and December 2015, were retrospectively enrolled. The SII was developed based on a training set of 197 patients from 2011 to 2013 and validated in an independent cohort of 222 patients from 2014 to 2015. Data on baseline clinicopathologic characteristics; pre-treatment laboratory variables such as absolute neutrophil, lymphocyte, and platelet counts; and carbohydrate antigen 19-9 (CA19-9), total bilirubin (TBIL), albumin (ALB), alkaline phosphatase (ALP), alanine transaminase (ALT), and aspartate transaminase (AST) levels were collected. The association between clinicopathologic characteristics and SII was assessed. The overall survival was calculated using the Kaplan-Meier survival curves and compared using the log-rank test. Univariate and multivariate Cox proportional hazard regression models were used to analyze the prognostic value of the SII.

Result: An optimal cutoff point for the SII of 440 stratified the patients with advanced pancreatic cancer into high $(>440)$ and low $(\leq 440)$ SII groups in the training cohort. Univariate and multivariate analyses revealed that the SII was an independent predictor for overall survival. The prognostic significance of the SII was confirmed in both normal and elevated CA19-9 levels.
\end{abstract}

Conclusion: The baseline SII serves as an independent prognostic marker for patients with advanced pancreatic cancer and can be used in patients with both normal and elevated CA19-9 levels.

Keywords: Pancreatic cancer, Prognostic marker, Systemic immune-inflammation index, CA19-9

\section{Background}

Pancreatic cancer is one of the most common malignant tumors and the seventh leading cause of cancer-related mortality worldwide [1]. In China, pancreatic cancer is the ninth type of cancer with the highest incidence and is ranked sixth in cancer-related mortality [2]. Surgical resection is the only curative treatment option; however,

\footnotetext{
*Correspondence: chengkll@sina.com

${ }^{1}$ Department of Integrative Oncology, Fudan University Shanghai Cancer Center, Shanghai 200032, China

Full list of author information is available at the end of the article
}

$80-85 \%$ of patients are diagnosed at advanced, inoperable stages [3]. Fluorouracil, leucovorin, irinotecan, and oxaliplatin (FOLFIRINOX) or gemcitabine-based chemotherapy is currently the standard treatment for these patients [4-6]. However, most patients do not respond or partially respond to the treatment, with a 5 -year survival rate of $<5 \%$ [7]. Therefore, identifying the prognostic marker that helps to predict survival and guides the optimal therapy in patients with advanced pancreatic cancer is crucial.

Tumor-promoting inflammation has been recognized as an enabling characteristic of cancer [8]. The interplay 
between local immune response and systemic inflammation plays vital roles in cancer progression and patient survival [9]. The inflammatory response can be represented by the level of neutrophils, lymphocytes, platelets, and acute-phase proteins. These parameters are simple and easy to measure using standardized assays in clinical practice. Recently, neutrophils, lymphocytes, and platelets have been used in a joined tool, a systemic immune-inflammation index (SII), to obtain the prognostic information in patients with various malignant tumors, such as hepatocellular carcinoma, esophageal squamous cell carcinoma, gastric cancer, non-small-cell lung cancer, and colorectal cancer [10-14]. Mohammad et al. reported that SII is an independent predictor of cancer-specific survival and recurrence in resectable pancreatic cancer [15]. However, the significance of SII as a prognostic predictor in patients with advanced pancreatic cancer has not been examined.

Carbohydrate antigen 19-9 (CA19-9) is an extensively studied and validated serum biomarker with multiple clinical application in pancreatic cancer. It has a sensitivity and specificity of $79-81 \%$ and $82-90 \%$ for diagnosis in symptomatic patients [16, 17]; but is not useful as a screening marker because of low positive predictive value $(0.5-0.9 \%)[18,19]$. CA19-9 also has prognostic and predictive value in resectable and advanced disease settings [20-23]. However, false negative results in Lewis negative genotype [24] and an increase false positive results in cases of biliary infection, inflammation, or biliary obstruction [25] may limit the prognostic role of serum CA19-9 in pancreatic cancer.

This study aimed to evaluate the prognostic value of SII in patients with advanced pancreatic cancer. Furthermore, based on different serum CA19-9 levels, the prognostic value of the SII was investigated separately.

\section{Patients and methods}

\section{Ethics statement}

This study was approved by the Ethics Committee of Fudan University Shanghai Cancer Center. All procedures were performed by the ethics standards of our institutional research committee and with those of the 1964 Helsinki Declaration and its later amendments or comparable ethical standards. Written informed consent was obtained from each participant by the institutional guidelines.

\section{Patient characteristics}

A retrospective cohort study consisting of 419 patients diagnosed with advanced pancreatic cancer from January 2011 to December 2015 was conducted. All the patients were diagnosed and received primary treatment at Fudan University Shanghai Cancer Center. Patients who met the following criteria were enrolled in this study. (1) patients histologically or cytologically confirmed to have pancreatic adenocarcinoma and not recommended for curative resection; (2) those with stage III and IV tumors according to the 8th edition of the American Joint Committee on Cancer (Chicago, IL, USA) [26]; (3) those with no other primary malignant tumors found during treatment; and (4) those who did not have any hematologic disorder. The exclusion criteria included a lack of complete clinicopathologic and follow-up data, tumors not originating from the pancreas, and acute inflammatory diseases.

Our analyses involved two independent patient cohorts: the training cohort consisting of 197 patients diagnosed from January 2011 to December 2013 and the validation cohort consisting of 222 patients diagnosed from January 2014 to December 2015. The primary endpoint of this study was overall survival (OS), which was defined as the interval between the diagnosis and death or the last follow-up. An independent researcher performed the follow-up work by conducting telephone interviews or reviewing medical records. Follow-up was terminated on December 31, 2016, in the training cohort and December 31, 2017, in the validation cohort.

\section{Clinical variables}

Data on clinical variables, including demographic data, complete blood counts, tumor location, stage, CA 19-9 levels, and liver function parameters, such as total bilirubin (TBIL), albumin (ALB), alkaline phosphatase (ALP), alanine transaminase (ALT), and aspartate transaminase (AST) were collected. All laboratory parameters were assayed during routine workups before cancer diagnostic interventions. Data were extracted from the Electronic Medical Record System of Fudan University Shanghai Cancer Center.

\section{Serum assays for CA19-9}

Baseline serum CA19-9 levels were examined within 1 week before the initiation diagnosis of pancreatic adenocarcinoma. Serum CA19-9 levels were detected using an electrochemiluminescence immunoassay on the Roche Cobas e601 immunoassay analyzer (Roche Diagnostics, Mannheim, Germany). Normal CA19-9 was defined as CA19-9 level lower than $37 \mathrm{U} / \mathrm{mL}$ [27].

\section{Systemic immune-inflammation index}

The SII is an index based on the platelet (P), neutrophil $(\mathrm{N})$, and lymphocyte $(\mathrm{L})$ counts and calculated using the following formula: $\mathrm{SII}=\mathrm{P}^{*} \mathrm{~N} / \mathrm{L}$ as defined previously [10].

The X-tile 3.6.1 software (Yale University, New Haven, CT) was used for bioinformatic analysis to determine the optimal cutoff value of SII in the training 
cohort [28]. Consequently, the SII scores were stratified into $\leq 440 * 10^{9}$ or $>440 * 10^{9}$ for all subsequent analyses.

\section{Statistical analysis}

Continuous variables were presented as the median and range. Cumulative survival rates were calculated using the Kaplan-Meier method, and between-group differences were assessed using the log-rank test. Univariate and multivariate analyses were calculated using the Cox proportional hazards regression model. Pearson's Chi square test was used to compare groups. A $P$ value of $<0.05$ was considered to be statistically significant.

\section{Results}

\section{Patient characteristics}

The clinical characteristics of patients in the training and validation cohorts are shown in Table 1 . For the whole study population, 269 (64.2\%) were men and 150 (35.8\%) women, with the median age of 61 (range 25-84) years. Additionally, 88 patients (27.5\%) were diagnosed with locally advanced disease, and the remaining 232 (72.5\%) were diagnosed with metastatic disease. In the training cohort, all patients died during the last follow-up, with a median OS of 6.6 months. In the validation cohort, six out of 219 patients survived during the last follow-up, with a median OS of 8.7 months. The SII of $>440$ was observed in $53.8 \%(106 / 197)$ of the patients in the training cohort and $59.9 \%(133 / 222)$ in the validation cohort. As shown in Table 1, the clinicopathologic characteristics were similar between the two cohorts.

\section{Association between the SII and clinicopathologic parameters}

The correlation between the SII and clinicopathologic parameters is shown in Table 2. In the training cohort, patients with an SII of $>440$ were more likely to have metastasis $(\mathrm{P}=0.014)$ and low ALB levels $(\mathrm{P}=0.023)$. In the validation cohort, an SII of $>440$ was associated with high ALP levels $(\mathrm{P}=0.016)$.

\section{Prognostic significance of the SII in the training cohort}

Univariate analysis indicated that metastasis, CA199, TBIL, and SII were prognostic factors for OS in the training cohort, whereas age, gender, tumor location, ALB, ALP, ALT, and AST had no prognostic value for OS (Table 3). A high SII was significantly associated with shorter OS (hazard ratio $[\mathrm{HR}]=1.549,95 \%$ confidence interval $[\mathrm{CI}]=1.16-2.06, \mathrm{P}=0.003)$.

The Kaplan-Meier analysis indicated that higher SII was associated with shorter OS $(\mathrm{P}=0.002$, Fig. 1a). The median OS was 7.9 months and 5.7 months for patients with SII of $\leq 440$ and SII of $>440$, respectively. Based on the result of our multivariate analysis, the SII was an
Table 1 The clinicopathologic characteristics of patients in the training and validation cohorts

\begin{tabular}{|c|c|c|c|c|c|}
\hline \multirow[t]{2}{*}{ Variables } & \multicolumn{2}{|l|}{ Training } & \multicolumn{2}{|c|}{ Validation } & \multirow[t]{2}{*}{$P$} \\
\hline & $N=197$ & $\%$ & $N=222$ & $\%$ & \\
\hline \multicolumn{6}{|l|}{ Age (years) } \\
\hline$\leq 60$ & 95 & 48.2 & 105 & 47.3 & \multirow[t]{2}{*}{0.922} \\
\hline$>60$ & 102 & 51.8 & 117 & 52.7 & \\
\hline \multicolumn{6}{|l|}{ Sex } \\
\hline Male & 122 & 61.9 & 147 & 66.2 & \multirow[t]{2}{*}{0.414} \\
\hline Female & 75 & 38.1 & 75 & 33.8 & \\
\hline \multicolumn{6}{|l|}{ Location } \\
\hline Head & 90 & 40.5 & 87 & 44.2 & \multirow[t]{2}{*}{0.488} \\
\hline Body/tail & 132 & 59.5 & 110 & 55.8 & \\
\hline \multicolumn{6}{|l|}{ Metastasis } \\
\hline No & 41 & 20.8 & 42 & 18.9 & \multirow[t]{2}{*}{0.713} \\
\hline Yes & 156 & 79.2 & 180 & 81.1 & \\
\hline \multicolumn{6}{|c|}{ CA19-9 (U/mL) } \\
\hline$\leq 37$ & 44 & 22.3 & 52 & 23.4 & \multirow[t]{2}{*}{0.817} \\
\hline$>37$ & 153 & 77.7 & 170 & 76.6 & \\
\hline \multicolumn{6}{|c|}{ TBIL ( $\mu \mathrm{mol} / \mathrm{L})$} \\
\hline$\leq 17$ & 162 & 82.2 & 184 & 82.9 & \multirow[t]{2}{*}{0.898} \\
\hline$>17$ & 35 & 17.8 & 38 & 17.1 & \\
\hline \multicolumn{6}{|l|}{ ALB (g/L) } \\
\hline$\leq 35$ & 13 & 6.6 & 13 & 5.9 & \multirow[t]{2}{*}{0.840} \\
\hline$>35$ & 184 & 93.4 & 209 & 94.1 & \\
\hline \multicolumn{6}{|l|}{ ALP (U/L) } \\
\hline$\leq 125$ & 139 & 70.6 & 157 & 70.7 & \multirow[t]{2}{*}{1.000} \\
\hline$>125$ & 58 & 29.4 & 65 & 29.3 & \\
\hline \multicolumn{6}{|l|}{$\mathrm{ALT}(\mathrm{U} / \mathrm{L})$} \\
\hline$\leq 35$ & 148 & 75.1 & 172 & 77.5 & \multirow[t]{2}{*}{0.645} \\
\hline$>35$ & 49 & 24.9 & 50 & 22.5 & \\
\hline \multicolumn{6}{|l|}{ AST (U/L) } \\
\hline$\leq 40$ & 165 & 83.8 & 186 & 83.8 & \multirow[t]{2}{*}{1.000} \\
\hline$>40$ & 32 & 16.2 & 36 & 16.2 & \\
\hline
\end{tabular}

$C A$ carbohydrate antigen, $T B I L$ total bilirubin, $A L B$ albumin, $A L P$ alkaline phosphatase, $A L T$ alanine transaminase, $A S T$ aspartate transaminase

independent prognostic factor for OS (HR $=1.502,95 \%$ $\mathrm{CI}=1.13-2.00, \mathrm{P}=0.005$, Table 4 ).

\section{Validation of the SII in an independent cohort}

The prognostic value of the SII was confirmed in an independent validation cohort of 222 patients. The results were similar with those obtained from the training cohort (Table 3). The Kaplan-Meier analysis showed that the high SII was associated with shorter OS in the validation cohort $(\mathrm{P}=0.007$, Fig. $1 \mathrm{~b})$ and total cohort $(\mathrm{P}<0.001$, Fig. 1c). Univariate and multivariate analyses demonstrated that the SII was significantly correlated with $\mathrm{OS}(\mathrm{HR}=1.455,95 \% \mathrm{CI}=1.10-1.92$, $\mathrm{P}=0.008$, Table 4). 
Table 2 The correlation between SII and clinicopathologic characteristics in training and validation cohorts

\begin{tabular}{|c|c|c|c|c|c|c|c|c|c|c|}
\hline \multirow[t]{2}{*}{ Variables } & \multicolumn{5}{|c|}{ Training $(N=197)$} & \multicolumn{5}{|c|}{ Validation $(\mathrm{N}=222)$} \\
\hline & $\mathrm{SII} \leq 440$ & $\%$ & $\mathrm{SII}>440$ & $\%$ & $\mathbf{P}$ & $\mathrm{SII} \leq 440$ & $\%$ & SII $>440$ & $\%$ & $\mathbf{P}$ \\
\hline \multicolumn{11}{|l|}{ Age (years) } \\
\hline$\leq 60$ & 39 & 42.9 & 56 & 52.8 & 0.198 & 41 & 46.1 & 64 & 48.1 & 0.785 \\
\hline$>60$ & 52 & 57.1 & 50 & 47.2 & & 48 & 53.9 & 69 & 51.9 & \\
\hline \multicolumn{11}{|l|}{ Sex } \\
\hline Male & 55 & 60.4 & 67 & 63.2 & 0.769 & 62 & 69.7 & 85 & 63.9 & 0.389 \\
\hline Female & 36 & 39.6 & 39 & 36.8 & & 27 & 30.3 & 48 & 36.1 & \\
\hline \multicolumn{11}{|l|}{ Location } \\
\hline Head & 34 & 37.4 & 53 & 50 & 0.085 & 38 & 42.7 & 52 & 39.1 & 0.676 \\
\hline Body/tail & 57 & 62.6 & 53 & 50 & & 51 & 57.3 & 81 & 60.9 & \\
\hline \multicolumn{11}{|l|}{ Metastasis } \\
\hline No & 26 & 28.6 & 15 & 14.2 & 0.014 & 19 & 21.3 & 23 & 17.3 & 0.487 \\
\hline Yes & 65 & 71.4 & 91 & 85.8 & & 70 & 78.7 & 110 & 82.7 & \\
\hline \multicolumn{11}{|c|}{ CA19-9 (U/mL) } \\
\hline$\leq 37$ & 22 & 50.0 & 22 & 50.0 & 0.609 & 22 & 42.3 & 30 & 57.7 & 0.748 \\
\hline$>37$ & 69 & 45.1 & 84 & 54.9 & & 67 & 39.4 & 103 & 60.6 & \\
\hline \multicolumn{11}{|c|}{ TBIL ( $\mu \mathrm{mol} / \mathrm{L})$} \\
\hline$\leq 17$ & 80 & 87.9 & 82 & 77.4 & 0.062 & 77 & 86.5 & 107 & 80.5 & 0.278 \\
\hline$>17$ & 11 & 12.1 & 24 & 22.6 & & 12 & 13.5 & 26 & 19.5 & \\
\hline \multicolumn{11}{|l|}{$A L B(g / L)$} \\
\hline$\leq 35$ & 2 & 2.2 & 11 & 10.4 & 0.023 & 5 & 5.6 & 8 & 6.0 & 1.000 \\
\hline$>35$ & 89 & 97.8 & 95 & 89.6 & & 84 & 94.4 & 125 & 94.0 & \\
\hline \multicolumn{11}{|l|}{$\mathrm{ALP}(\mathrm{U} / \mathrm{L})$} \\
\hline$\leq 125$ & 70 & 76.9 & 69 & 65.1 & 0.085 & 71 & 79.8 & 86 & 64.7 & 0.016 \\
\hline$>125$ & 21 & 23.1 & 37 & 34.9 & & 18 & 20.2 & 47 & 35.3 & \\
\hline \multicolumn{11}{|l|}{$\operatorname{ALT}(\mathrm{U} / \mathrm{L})$} \\
\hline$\leq 35$ & 69 & 75.8 & 79 & 74.5 & 0.870 & 72 & 80.9 & 100 & 75.2 & 0.332 \\
\hline$>35$ & 22 & 24.2 & 27 & 25.5 & & 17 & 19.1 & 33 & 24.8 & \\
\hline \multicolumn{11}{|l|}{ AST (U/L) } \\
\hline$\leq 40$ & 76 & 83.5 & 89 & 84.0 & 1.000 & 80 & 89.9 & 106 & 79.7 & 0.062 \\
\hline$>40$ & 15 & 16.5 & 17 & 16.0 & & 9 & 10.1 & 27 & 20.3 & \\
\hline
\end{tabular}

$C A$ carbohydrate antigen, $T B I L$ total bilirubin, $A L B$ albumin, $A L P$ alkaline phosphatase, $A L T$ alanine transaminase, $A S T$ aspartate transaminase

\section{Prognostic significance of the SII in patients with normal and elevated CA19-9 levels}

We further investigated the prognostic significance of the SII according to different CA19-9 levels. We found that the SII score was significantly correlated with OS in both normal and elevated CA19-9 groups in the training cohort $(\mathrm{P}=0.028$, Fig. $2 \mathrm{a}$ and $\mathrm{P}=0.042$, Fig. $2 \mathrm{~b})$. The prognostic value for OS in normal and elevated CA19-9 groups was also confirmed in the validation cohort $(P=0.026$, Fig. $2 c$ and $P=0.037$, Fig. $2 d)$ and total cohorts $(P=0.002$, Fig. 2e and $P=0.006$, Fig. 2f $)$.

\section{Discussion}

Systemic inflammation is an essential promoter of proliferation, invasion, and metastasis of tumor cells $[29,30]$. The immune system also plays a vital role in cancer surveillance and elimination [31]. In this study, we constructed an immune-inflammation-based prognostic score (SII) based on the peripheral lymphocyte, neutrophil, and platelet counts. Furthermore, we demonstrated SII as a predictor of survival in patients with advanced pancreatic cancer in two independent cohorts. Also, the SII maintained its prognostic significance in both normal and elevated CA19-9 levels.

The relationship between SII and advanced pancreatic cancer prognosis may be due to the high SII results from thrombocythemia, neutrophilia, and lymphopenia, which suggests an elevated inflammatory status and decreased immune system response. Cancer inflammation has been known to have a normal impact on survival [31, 32]. Increasing evidence has shown 
Table 3 Univariate Cox regression analyses of the SII with clinicopathologic characteristics [training cohort $(n=197)$ and validation cohort $(n=222)]$

\begin{tabular}{|c|c|c|c|c|}
\hline \multirow[t]{2}{*}{ Variables } & \multicolumn{2}{|l|}{ Training } & \multicolumn{2}{|l|}{ Validation } \\
\hline & HR $(95 \% \mathrm{Cl})$ & $\mathbf{P}$ & $\mathrm{HR}(95 \% \mathrm{Cl})$ & $\mathbf{P}$ \\
\hline Age, years ( $\leq 60$ vs. $>60)$ & $1.238(0.93-1.64)$ & 0.139 & $0.907(0.69-1.19)$ & 0.474 \\
\hline Sex (male vs. female) & $1.234(0.92-1.65)$ & 0.154 & $1.011(0.76-1.34)$ & 0.942 \\
\hline Location (head vs. body/tail) & $0.845(0.64-1.12)$ & 0.243 & $0.933(0.71-1.23)$ & 0.620 \\
\hline Metastasis (no vs. yes) & $1.580(1.11-2.26)$ & 0.012 & $1.686(1.18-2.41)$ & 0.004 \\
\hline CA19-9, U/mL ( $\leq 37$ vs. > 37) & $1.580(1.12-2.23)$ & 0.009 & $1.191(0.87-1.64)$ & 0.285 \\
\hline TBIL, $\mu \mathrm{mol} / \mathrm{L}(\leq 17$ vs. $>17)$ & $1.578(1.09-2.28)$ & 0.015 & $1.472(1.03-2.10)$ & 0.033 \\
\hline ALB, g/L ( $\leq 35$ vs. $>35)$ & $0.722(0.41-1.27)$ & 0.259 & $0.644(0.36-1.16)$ & 0.140 \\
\hline $\mathrm{ALP}, \mathrm{U} / \mathrm{L}(\leq 125 \mathrm{vs} .>125)$ & $1.101(0.81-1.50)$ & 0.540 & $1.272(0.94-1.72)$ & 0.118 \\
\hline $\mathrm{ALT}, \mathrm{U} / \mathrm{L}(\leq 35 \mathrm{vs.}>35)$ & $1.061(0.77-1.47)$ & 0.719 & $1.090(0.79-1.50)$ & 0.598 \\
\hline AST, U/L ( $\leq 40$ vs. $>40)$ & $1.019(0.70-1.49)$ & 0.922 & $1.437(1.00-2.07)$ & 0.052 \\
\hline SII ( $\leq 440$ vs. $>440)$ & $1.549(1.16-2.06)$ & 0.003 & $1.458(1.11-1.92)$ & 0.008 \\
\hline
\end{tabular}

$C A$ carbohydrate antigen, $T B I L$ total bilirubin, $A L B$ albumin, $A L P$ alkaline phosphatase, $A L T$ alanine transaminase, $A S T$ aspartate transaminase, SII systemic immuneinflammation index
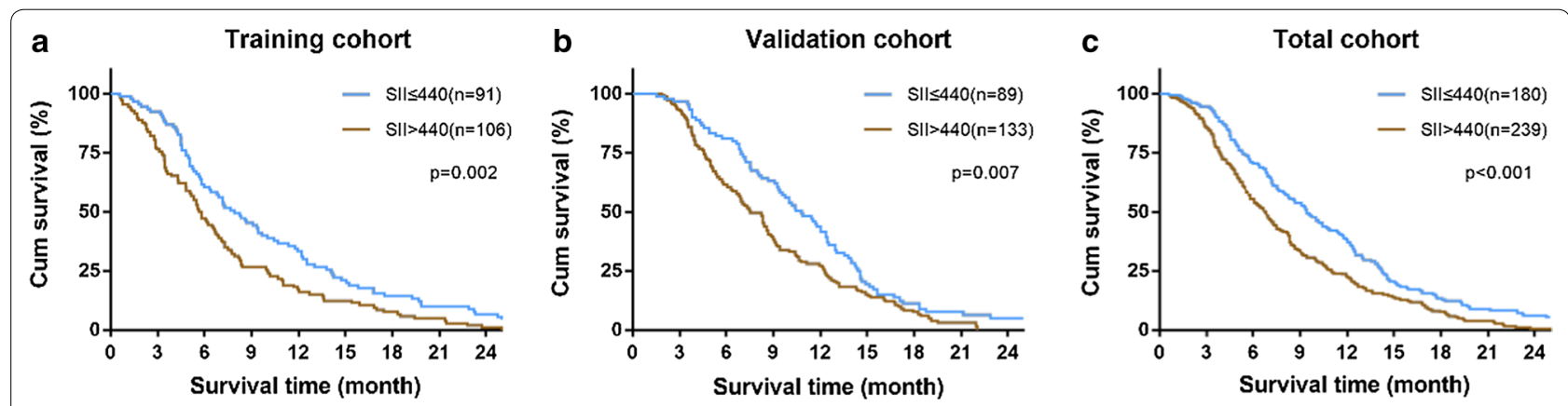

Fig. 1 Prognostic significance of SII in patients with advanced pancreatic cancer. Kaplan-Meier analysis of OS for SII in the training (a), validation (b), and total cohorts (c)

Table 4 Multivariate Cox regression analyses of the SII in the training and validation cohorts

\begin{tabular}{|c|c|c|c|c|}
\hline \multirow[t]{2}{*}{ Variables } & \multicolumn{2}{|l|}{ Training } & \multicolumn{2}{|l|}{ Validation } \\
\hline & $\mathrm{HR}(95 \% \mathrm{Cl})$ & $\mathbf{P}$ & $\mathrm{HR}(95 \% \mathrm{Cl})$ & $\mathbf{P}$ \\
\hline Metastasis (no vs. yes) & $1.318(0.91-1.91)$ & 0.144 & $1.696(1.19-2.42)$ & 0.004 \\
\hline CA19-9, U/mL ( $\leq 37$ vs. $>37)$ & $1.521(1.08-2.15)$ & 0.017 & NA & NA \\
\hline TBIL, $\mu \mathrm{mol} / \mathrm{L}(\leq 17$ vs. $>17)$ & $1.325(0.91-1.93)$ & 0.145 & $1.509(1.06-2.16)$ & 0.024 \\
\hline $\mathrm{SII}(\leq 440$ vs. $>440)$ & $1.502(1.13-2.00)$ & 0.005 & $1.455(1.10-1.92)$ & 0.008 \\
\hline
\end{tabular}

CA carbohydrate antigen, $T B I L$ total bilirubin, SII systemic immune-inflammation index

that neutrophilia and thrombocythemia are associated with pro-cancer effects [33-36]. Neutrophils can not only enhance cancer cell invasion, proliferation, and metastasis but also assist the evasion of cancer cells on immune surveillance [37]. Platelets can guard tumor cells against immune elimination and promote their arrest at the endothelium, supporting the establishment of secondary lesions [38]. For example, in pancreatic cancer, platelets support the adhesion of tumor cells to escape from the host's immune surveillance. Circulating tumor cells (CTCs) are neoplastic cells shed from a solid tumor into the bloodstream and associated with tumor metastases [39] [40]. Platelets can also protect the CTCs from shear stresses during circulation, 


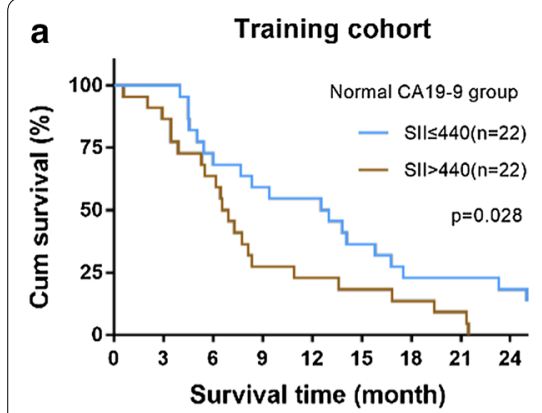

b

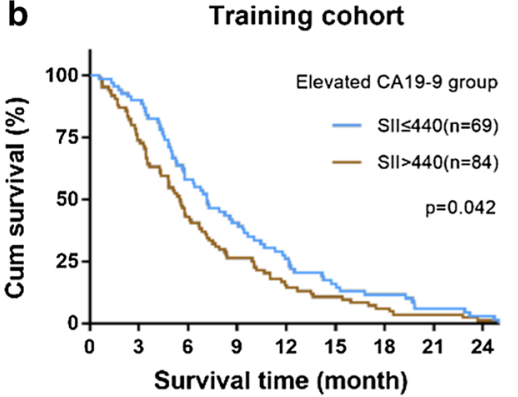

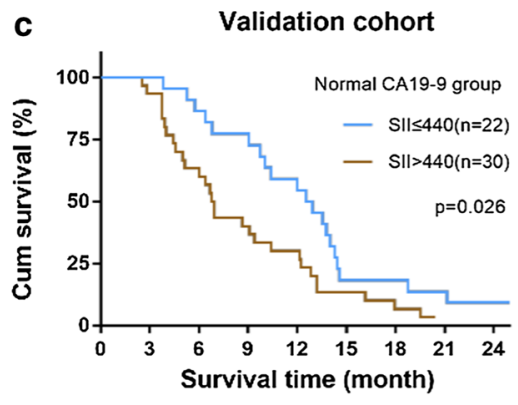

d

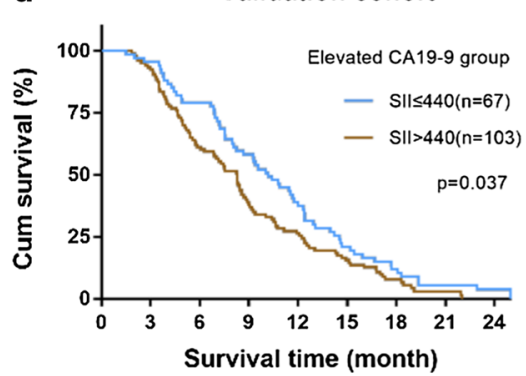

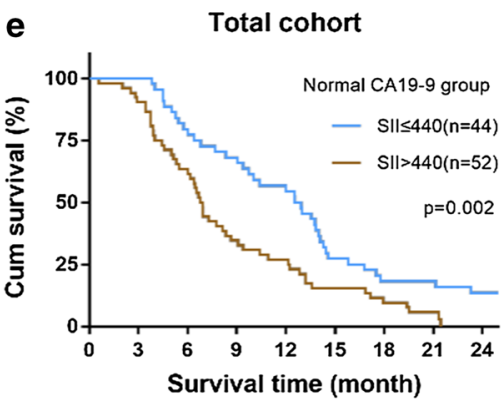

f

Total cohort

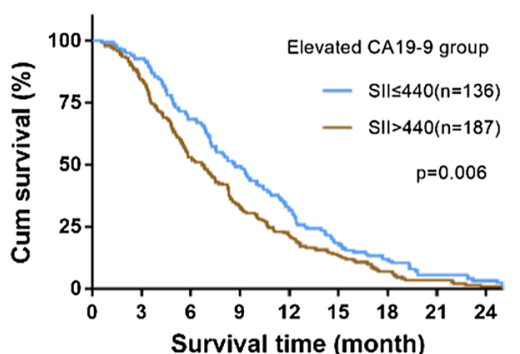

Fig. 2 Prognostic significance of SII in patients with normal and elevated CA19-9. Kaplan-Meier analysis of OS for SII in normal and elevated CA19-9 groups in the training $(\mathbf{a}, \mathbf{b})$, validation $(\mathbf{c}, \mathbf{d})$, and total cohorts $(\mathbf{e}, \mathbf{f})$

inducing CTC epithelial-mesenchymal transition [41]. Our results showed that patients with high SII in the training cohort were more likely to have metastatic disease.

Conversely, lymphocytes are known to play a crucial role in tumor defense by inducing cell death and inhibiting cell proliferation and migration [42]. Lymphopenia, which indicates the ineffectiveness of the immune surveillance systems, is also observed in pancreatic cancer [43] and has been reportedly associated with poor survival in several malignant tumors [44]. Considering these reasons, patients with higher SII presented with poor survival. These results help us to better understand the role of neutrophils, platelets, and lymphocytes in cancer and their relationship with immunity and inflammation. Also, patients with advanced pancreatic cancer who have a high SII might benefit from targeted anti-inflammatory agents, such as aspirin [45]. Recently, immune checkpoint inhibitors have been approved for the treatment of various cancer, which urges the development of immunespecific biomarkers. Previous studies have reported the prognostic value of SII in patients undergoing immunotherapy [46]. Therefore, the SII may serve as a prognostic marker in immunotherapy.

CA19-9 is a sialylated Lewis A blood group antigen and commonly expressed and shed in pancreatic and hepatobiliary disease and in many malignancies.
Although CA19-9 is a well-established serum biomarker to predict the prognosis of pancreatic cancer $[47,48]$, it is important to note that CA19-9 may be undetectable in Lewis antigen-negative individuals [24]. In our study, approximately a quarter of the patients with advanced pancreatic cancer presented with normal CA19-9 levels $(\leq 37 \mathrm{U} / \mathrm{mL})$ upon the diagnosis. Furthermore, CA19-9 may be falsely positive in cases of biliary infection or biliary obstruction [25]. In our study, about $17 \%$ patients presented with high total bilirubin $(>17 \mu \mathrm{mol} / \mathrm{L})$. In addition, the level of CA19-9 was also influenced by age and gender [49]. In consequence, the prognostic value of CA19-9 in pancreatic cancer is limited. Our results showed that SII serves as an independent prognostic factor in not only normal CA19-9 group but also elevated CA19-9 group. This suggests that SII complements with CA19-9 in predicting the outcomes in patients with advanced pancreatic cancer.

This study has a few limitations. First, this was a retrospective study performed in a single center; thus, multicenter studies should be performed to provide stronger evidence. Second, further studies should be conducted to explore the underlying mechanism between SII and cancer biology. Third, although the prognostic value of the SII was confirmed, we did not compare the discrimination ability of the SII with other prognostic markers. 


\section{Conclusion}

In conclusion, 419 patients with advanced pancreatic cancer were retrospectively enrolled, and the prognostic significance of the SII was determined in two independent cohorts. Our result confirmed that the SII could serve as an independent prognostic marker in patients with advanced pancreatic cancer. Also, the SII can be used in patients with both normal and elevated CA19-9 levels.

\begin{abstract}
Abbreviations
SII: systemic immune-inflammation index; CA19-9: carbohydrate antigen 19-9; TBIL: total bilirubin; ALB: albumin; ALP: alkaline phosphatase; ALT: alanine transaminase; AST: aspartate transaminase; OS: overall survival; HR: hazard ratio; $\mathrm{Cl}$ : confidence interval.
\end{abstract}

\section{Authors' contributions}

KZ: data acquisition and manuscript writing, YQH and DW: data acquisition and data analysis, LYC and CJW: follow up and quality control of data, ZC: manuscript review, LML and HC: study concept and design. All authors read and approved the final manuscript.

\section{Author details}

${ }^{1}$ Department of Integrative Oncology, Fudan University Shanghai Cancer Center, Shanghai 200032, China. ${ }^{2}$ Department of Oncology, Shanghai Medical College, Fudan University, Shanghai 200032, China.

\section{Acknowledgements}

The authors acknowledge Xiu-Mei Zhang for her assistant in follow-up work.

\section{Competing interests}

The authors declare that they have no competing interests.

\section{Availability of data and materials}

All data included in the present study were presented in the main manuscript.

\section{Consent for publication}

Informed consent was obtained from all participants for publication.

\section{Ethics approval and consent to participate}

This study was approved by the Ethics Committee of Fudan University Shanghai Cancer Center. All procedures were performed by the ethical standards of our institutional research committee and with those of the 1964 Helsinki Declaration and its later amendments or comparable ethical standards. Written informed consent was obtained from each participant by the institutional guidelines.

\section{Funding}

This study was supported by the National Nature Science Foundation of China (Grant Nos. 81673749, 81673746, and 81603439), the Development Action Plan of T.C.M. of the Shanghai Municipal Commission of Health and Family Planning (Grant Nos. ZY3-CCCX-3-3031 and ZY3-LCPT-2-1001), the Medical Guide Project of the Shanghai Municipal Commission for Science and Technology (Grant No. 15401932400), and the 'Xinglin Star' plan of the Shanghai Municipal Commission of Health and Family Planning (Grant No. ZY3-RCPY-2-2023).

\section{Publisher's Note}

Springer Nature remains neutral with regard to jurisdictional claims in published maps and institutional affiliations.

Received: 7 November 2018 Accepted: 11 January 2019

Published online: 18 January 2019

\section{References}

1. Bray F, Ferlay J, Soerjomataram I, Siegel R, Torre L, Jemal A. Global cancer statistics 2018: GLOBOCAN estimates of incidence and mortality worldwide for 36 cancers in 185 countries. CA Cancer J Clin. 2018;68(6):394-424.

2. Chen W, Zheng R, Baade PD, Zhang S, Zeng H, Bray F, et al. Cancer statistics in China. CA Cancer J Clin. 2016;66:115-32.

3. Kamisawa T, Wood LD, Itoi T, Takaori K. Pancreatic cancer. Lancet (London, England). 2016;388:73-85.

4. Burris HA, Moore MJ, Andersen J, Green MR, Rothenberg ML, Modiano MR, et al. Improvements in survival and clinical benefit with gemcitabine as first-line therapy for patients with advanced pancreas cancer: a randomized trial. J Clin Oncol. 1997;15:2403-13. https://doi.org/10.1200/ JCO.1997.15.6.2403.

5. Conroy T, Desseigne F, Ychou M, Bouché O, Guimbaud R, Bécouarn Y, et al. FOLFIRINOX versus gemcitabine for metastatic pancreatic cancer. N Engl J Med. 2011;364:1817-25. https://doi.org/10.1056/NEJMoa1011923.

6. Von Hoff DD, Ervin T, Arena FP, Chiorean EG, Infante J, Moore M, et al. Increased survival in pancreatic cancer with nab-paclitaxel plus gemcitabine. N Engl J Med. 2013;369:1691-703. https://doi.org/10.1056/NEJMo a1304369.

7. Miller KD, Siegel RL, Lin CC, Mariotto AB, Kramer JL, Rowland JH, et al. Cancer treatment and survivorship statistics, 2016. CA Cancer J Clin. 2016;66:271-89. https://doi.org/10.3322/caac.21349.

8. Hanahan D, Weinberg RA. Hallmarks of Cancer: the next generation. Cell. 2011;144:646-74. https://doi.org/10.1016/j.cell.2011.02.013.

9. Diakos Cl, Charles KA, McMillan DC, Clarke SJ. Cancer-related inflammation and treatment effectiveness. Lancet Oncol. 2014;15:e493-503. https ://doi.org/10.1016/S1470-2045(14)70263-3.

10. Hu B, Yang XR, Xu Y, Sun YF, Sun C, Guo W, et al. Systemic immune-inflammation index predicts prognosis of patients after curative resection for hepatocellular carcinoma. Clin Cancer Res. 2014;20:6212-22.

11. Wang L, Wang C, Wang J, Huang $X$, Cheng Y. A novel systemic immuneinflammation index predicts survival and quality of life of patients after curative resection for esophageal squamous cell carcinoma. J Cancer Res Clin Oncol. 2017;143:2077-86.

12. Chen L, Yan Y, Zhu L, Cong X, Li S, Song S, Song H, Xue Y, et al. Systemic immune-inflammation index as a useful prognostic indicator predicts survival in patients with advanced gastric cancer treated with neoadjuvant chemotherapy. Cancer Manag Res. 2017;9:849-67. https://doi. org/10.2147/cmar.s151026.

13. Tong $Y-S$, Tan J, Zhou X-L, Song Y-Q, Song Y-J. Systemic immune-inflammation index predicting chemoradiation resistance and poor outcome in patients with stage III non-small cell lung cancer. J Transl Med. 2017;15:221. https://doi.org/10.1186/s12967-017-1326-1.

14. Xie Q-K, Chen P, Hu W-M, Sun P, He W-Z, Jiang C, et al. The systemic immune-inflammation index is an independent predictor of survival for metastatic colorectal cancer and its association with the lymphocytic response to the tumor. J Transl Med. 2018;16:273. https://doi.org/10.1186/ s12967-018-1638-9.

15. Aziz MH, Sideras K, Aziz NA, Mauff K, Haen R, Roos D, et al. The systemicimmune-inflammation index independently predicts survival and recurrence in resectable pancreatic cancer and its prognostic value depends on bilirubin levels. Ann Surg. 2018. https://doi.org/10.1097/sla.00000 00000002660.

16. Satake K, Takeuchi T. Comparison of CA19-9 with other tumor markers in the diagnosis of cancer of the pancreas. Pancreas. 1994;9:720-4.

17. Huang Z, Liu F. Diagnostic value of serum carbohydrate antigen 19-9 in pancreatic cancer: a meta-analysis. Tumour Biol. 2014;35:7459-65.

18. Kim JE, Lee KT, Lee JK, Paik SW, Rhee JC, Choi KW. Clinical usefulness of carbohydrate antigen 19-9 as a screening test for pancreatic cancer in an asymptomatic population. J Gastroenterol Hepatol. 2004;19:182-6.

19. Ballehaninna UK, Chamberlain RS. The clinical utility of serum CA 19-9 in the diagnosis, prognosis and management of pancreatic adenocarcinoma: an evidence based appraisal. J Gastrointest Oncol. 2012;3:105-19.

20. Ferrone CR, Finkelstein DM, Thayer SP, Muzikansky A, Fernandez-delCastillo C, Warshaw AL. Perioperative CA19-9 levels can predict stage and survival in patients with resectable pancreatic adenocarcinoma. J Clin Oncol. 2006;24:2897-902. 
21. Hartwig W, Strobel O, Hinz U, Fritz S, Hackert T, Roth C, et al. CA19-9 in potentially resectable pancreatic cancer: perspective to adjust surgical and perioperative therapy. Ann Surg Oncol. 2013;20:2188-96.

22. Bauer TM, El-Rayes BF, Li X, Hammad N, Philip PA, Shields AF, et al. Carbohydrate antigen 19-9 is a prognostic and predictive biomarker in patients with advanced pancreatic cancer who receive gemcitabine-containing chemotherapy: a pooled analysis of 6 prospective trials. Cancer. 2013;119:285-92.

23. Hess V, Glimelius B, Grawe P, Dietrich D, Bodoky G, Ruhstaller T, et al. CA 19-9 tumour-marker response to chemotherapy in patients with advanced pancreatic cancer enrolled in a randomised controlled trial. Lancet Oncol. 2008;9:132-8.

24. Tempero MA, Uchida E, Takasaki H, Burnett DA, Steplewski Z, Pour PM. Relationship of carbohydrate antigen 19-9 and Lewis antigens in pancreatic cancer. Cancer Res. 1987;47:5501-3.

25. Mann DV, Edwards R, Ho S, Lau WY, Glazer G. Elevated tumour marker CA19-9: clinical interpretation and influence of obstructive jaundice. Eur J Surg Oncol. 2000;26:474-9.

26. Amin MB, Greene FL, Edge SB, Compton CC, Gershenwald JE, Brookland RK, et al. The Eighth Edition AJCC Cancer Staging Manual: Continuing to build a bridge from a population-based to a more "personalized" approach to cancer staging. CA: a cancer journal for clinicians. 2017;67:93-9.

27. Safi F, Beger HG, Bittner R, Buchler M, Krautzberger W. CA 19-9 and pancreatic adenocarcinoma. Cancer. 1986;57:779-83.

28. Camp RL, Dolled-Filhart M, Rimm DL. X-tile: a new bio-informatics tool for biomarker assessment and outcome-based cut-point optimization. Clin Cancer Res. 2004;10:7252-9.

29. Elinav E, Nowarski R, Thaiss CA, Hu B, Jin C, Flavell RA. Inflammationinduced cancer: crosstalk between tumours, immune cells and microorganisms. Nat Rev Cancer. 2013;13:759-71.

30. Candido J, Hagemann T. Cancer-related inflammation. J Clin Immunol. 2013;33(Suppl 1):S79-84.

31. Zou W. Immunosuppressive networks in the tumour environment and their therapeutic relevance. Nat Rev Cancer. 2005;5:263-74. https://doi. org/10.1038/nrc1586.

32. Mantovani A, Allavena P, Sica A, Balkwill F. Cancer-related inflammation. Nature. 2008;454:436-44.

33. Ocana A, Nieto-Jimenez C, Pandiella A, Templeton AJ. Neutrophils in cancer: prognostic role and therapeutic strategies. Mol Cancer. 2017;16:137.

34. Treffers LW, Hiemstra IH, Kuijpers TW, van den Berg TK, Matlung HL. Neutrophils in cancer. Immunol Rev. 2016;273:312-28.
35. Bambace NM, Holmes CE. The platelet contribution to cancer progression. J Thromb Haemost. 2011;9:237-49.

36. Gay LJ, Felding-Habermann B. Contribution of platelets to tumour metastasis. Nat Rev Cancer. 2011;11:123-34.

37. Mantovani A, Cassatella MA, Costantini C, Jaillon S. Neutrophils in the activation and regulation of innate and adaptive immunity. Nat Rev Immunol. 2011;11:519-31.

38. Stegner D, Dutting S, Nieswandt B. Mechanistic explanation for platelet contribution to cancer metastasis. Thromb Res. 2014;133(Suppl):S149-57.

39. Cristofanilli M, Budd GT, Ellis MJ, Stopeck A, Matera J, Miller MC, et al. Circulating tumor cells, disease progression, and survival in metastatic breast cancer. N Engl J Med. 2004;351:781-91.

40. Micalizzi DS, Maheswaran S, Haber DA. A conduit to metastasis: circulating tumor cell biology. Genes Dev. 2017;31:1827-40.

41. Labelle M, Begum S, Hynes RO. Direct signaling between platelets and cancer cells induces an epithelial-mesenchymal-like transition and promotes metastasis. Cancer Cell. 2011;20:576-90.

42. Coussens LM, Werb Z. Inflammation and cancer. Nature. 2002;420:860-7.

43. Sideras K, Braat H, Kwekkeboom J, van Eijck CH, Peppelenbosch MP, Sleijfer $\mathrm{S}$, et al. Role of the immune system in pancreatic cancer progression and immune modulating treatment strategies. Cancer Treat Rev. 2014;40:513-22. https://doi.org/10.1016/j.ctrv.2013.11.005.

44. Ray-Coquard I, Cropet C, Van Glabbeke M, Sebban C, Le Cesne A, Judson I, et al. Lymphopenia as a prognostic factor for overall survival in advanced carcinomas, sarcomas, and lymphomas. Cancer Res. 2009;69:5383-91.

45. Jiang M-J, Dai J-J, Gu D-N, Huang Q, Tian L. Aspirin in pancreatic cancer: chemopreventive effects and therapeutic potentials. Biochim Biophys Acta. 2016;1866:163-76.

46. Chovanec M, Cierna Z, Miskovska V, Machalekova K, Kalavska K, Rejlekova $\mathrm{K}$, et al. Systemic immune-inflammation index in germ-cell tumours. Br J Cancer. 2017;2018(118):1-8. https://doi.org/10.1038/bjc.2017.460.

47. Humphris JL, Chang DK, Johns AL, Scarlett CJ, Pajic M, Jones MD, et al. The prognostic and predictive value of serum CA19.9 in pancreatic cancer. Ann Oncol. 2012;23:1713-22.

48. Poruk KE, Gay DZ, Brown K, Mulvihill JD, Boucher KM, Scaife CL, et al. The clinical utility of CA 19-9 in pancreatic adenocarcinoma: diagnostic and prognostic updates. Curr Mol Med. 2013;13:340-51.

49. Wang Y-Z, Zhou Y-W, Xie X-Y, Li G-L, Wang X-H, Li F-Y, et al. The levels of carbohydrate antigen $19-9$ are associated with gender and age in Chinese population. Clin Lab. 2013;59:813-7.
Ready to submit your research? Choose BMC and benefit from:

- fast, convenient online submission

- thorough peer review by experienced researchers in your field

- rapid publication on acceptance

- support for research data, including large and complex data types

- gold Open Access which fosters wider collaboration and increased citations

- maximum visibility for your research: over 100M website views per year

At BMC, research is always in progress.

Learn more biomedcentral.com/submissions 